\title{
In Situ Lorentz Differential Phase Contrast STEM Characterization of Rashba Interaction on Skyrmion Thin Films
}

\author{
B. D. Esser ${ }^{1}$, A. S. Ahmed ${ }^{2}$, K. Meng ${ }^{2}$, J. Rowland ${ }^{2}$, M. Randeria ${ }^{2}$, R. K. Kawakami ${ }^{2}$, F. Y. Yang ${ }^{2}$ and \\ D. W. McComb ${ }^{1}$ \\ 1. Center for Electron Microscopy and Analysis, The Ohio State University, Columbus, OH. \\ 2. Department of Physics, The Ohio State University, Columbus, OH.
}

With a growing demand for ultra-high-density data storage and more energy-efficient electronic devices, new magnetic materials and device designs are of critical importance. Theory and experiment both show that materials with the skyrmion phase are potential candidates for next generation storage and computation because they are robust to defects due to their topological protection and because their size can be tuned to less than 10 nanometers in diameter. [1-6] Skyrmions are stabilized by competing interactions: the exchange interaction $(\mathrm{J})$ and the Dzyaloshinskii-Moriya (DM) interaction. The exchange interaction takes the form of a dot product $\left[-J S_{i} \cdot S_{j}\right]$ and is minimized by a parallel spin configuration; whereas, the DM interaction results in canted spins because it takes the form of a cross product $\left[D \cdot\left(S_{i} \times S_{j}\right)\right]$. The DM interaction can be further broken down into contributions from either broken bulk inversion symmetry (Dresselhaus DM) or broken mirror symmetry, such as at an interface (Rashba DM). Materials with purely Dresselhaus DM interaction such as the FeGe and MnSi B20 crystals (space group P2 13 ) form Bloch skyrmions, which are vortex-like, with spins canting and twisting away from the core of the skyrmion. In systems with pure Rashba DM interaction such as ultrathin metallic films and perovskite heterostructures, Néel skyrmions form with the so-called hedgehog-like structure where spins cant in a radially symmetry fashion away from the core of the skyrmion. It has been theoretically predicted that a combination of Dresselhaus and Rashba DM interaction will produce a skyrmion with mixed Bloch and Néel character, though these have yet to be experimentally observed. [7]

Experimentally, Bloch skyrmions have been observed in a number of systems using conventional Lorentz transmission electron microscopy (LTEM) and in situ cooling and variable magnetic field. The magnetic character of Néel skyrmions, on the other hand, makes LTEM imaging difficult without large sample tilts due to the lack of significant magnetic contrast. [8] To image Néel and potentially mixed Néel/Bloch skyrmions, we use in situ Lorentz differential phase contrast scanning transmission electron microscopy with variable applied magnetic field and temperatures between $90 \mathrm{~K}$ and room temperature.

In this work we study two systems: FeGe capped with various metallic films and bilayer $\mathrm{SrIrO}_{3} / \mathrm{SrRuO}_{3}$. The FeGe system inherently exhibits Bloch skyrmions because of its noncentrosymmetric crystal structure; however, the addition of a metallic thin film cap adds a Rashba DM interaction, the magnitude of which is dependent on the strength of the spin-orbit coupling of the metallic film, which in turn is roughly dependent on $\mathrm{Z}^{4}$. The proximity of the metallic thin film, and thus Rashba interaction, is predicted to make skyrmions more stable than in pure FeGe. In the latter system, bilayer perovskite $\mathrm{SrIrO}_{3} / \mathrm{SrRuO}_{3}$ is grown on $\mathrm{SrTiO}_{3}$ via off-axis magnetron sputtering, as seen in Figure 1. The perovskite system has only the Rashba DM interaction with a contribution of large spinorbit coupling from the Ir, forming Néel skyrmions with a diameter of roughly 6 nanometers as predicted by the presence of a large topological hall signal, which is typically used as indirect evidence 
of skyrmions. In both systems, the effect of structural and compositional inhomogeneities is also studied using high spatial resolution aberration corrected STEM and energy dispersive x-ray spectroscopy [9].

\section{References:}

[1] J. Sampaio et al, Nature Nanotechnology 8 (2013), p. 839.

[2] R. Tomasello et al, Scientific Reports 4 (2014), p. 6784.

[3] G. Yu et al, Nano Letters 16 (2016), p. 1981.

[4] X.Z. Yu et al, Nature Communications 3 (2012), p. 988.

[5] X. Zhang et al, Scientific Reports 5 (2015), p. 9400.

[6] X. Zhang et al, Scientific Reports 5 (2015), p. 7643.

[7] A.S. Ahmed et al, Journal of Crystal Growth 467 (2017), p. 38.

[8] S.D. Pollard et al, Nature Communications 8 (2017), p. 14761.

[9] This work was supported in part by The Ohio State University Materials Research Seed Grant Program, funded by the Center for Emergent Materials, an NSF-MRSEC, grant DMR-1420451, the Center for Exploration of Novel Complex Materials, and the Institute for Materials Research, as well as from the Ohio State Materials Seed Grants (MTB-G00010 and MTB-G00012).

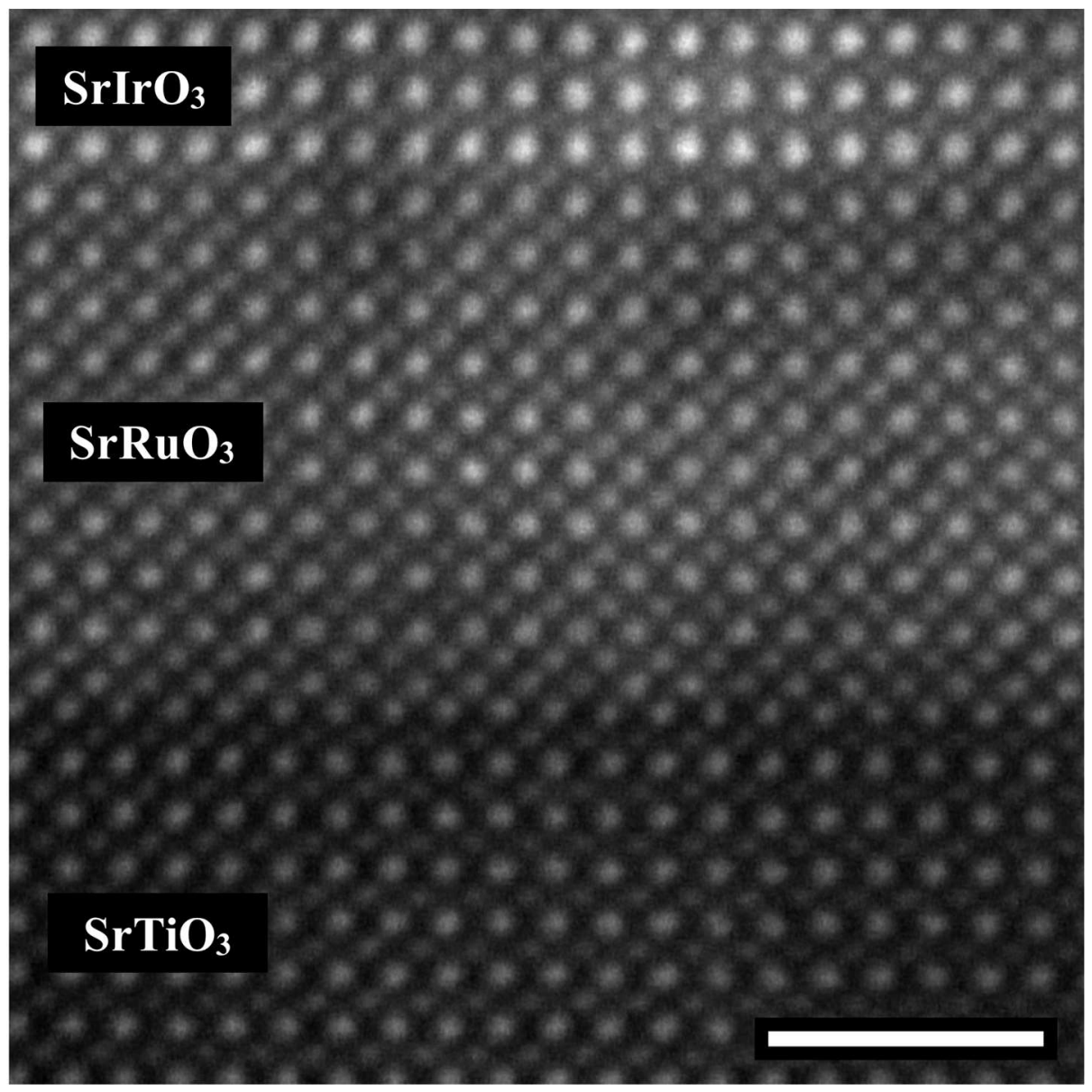

Figure 1. High angle annular dark field STEM image of (2 unit cell) $\mathrm{SrIrO}_{3} /$ (10 unit cell) $\mathrm{SrRuO}_{3}$ bilayer grown on $\mathrm{SrTiO}_{3}$ via off-axis magnetron sputtering. Scale bar is $2 \mathrm{~nm}$. 